\title{
On a Stability Theorem for Local Uniformization in Characteristic $p^{*}$
}

By

\author{
T. T. $\mathrm{MOH}^{* *}$
}

\begin{abstract}
A 2nd numerical $d$ is bounded under blow-ups.

\section{Introduction}

In [H, p. 123] Prof. H. Hironaka pointed out: "The point is that the associated Tschirnhausen polynomial undergoes the same law of transformation as the original polynomial under permissible blow-ups". For the notion of Tschirnhausen polynomials, or equivalently the approximate roots, the reader is referred to $[\mathbb{A}-\mathbb{M} \mathbb{1} \& 2],[\mathbb{M}]$ or [H]. As established by Prof. H. Hironaka the local uniformization problem in characteristic $p>0$ is to use monoidal transformations to resolve the singularity of an algebroid equation of the following form over $k\left[\left[x_{1}, \ldots, x_{n}\right]\right]$

$$
z^{p^{e}}+\sum_{i=1}^{p^{e}} f_{i}\left(x_{1}, \ldots, x_{n}\right) z^{p^{e}-i}=0 \text { with ord }\left(f_{i}\right) \geq i \text {. }
$$

A specially important case is the following purely inseparable equation which is the topic of this article,

$$
z^{p^{e}}+f_{p^{e}}\left(x_{1}, \ldots, x_{n}\right)=0
$$

where $f_{p^{e}}\left(x_{1}, \ldots, x_{n}\right) \in k\left[\left[x_{1}, \ldots, x_{n}\right]\right]$ and $f_{p^{e}}(0, \ldots, 0)=0$. After some monoidal transformation the above equation will be transformed to

$$
z^{p^{e}}+\left(\prod_{i=1}^{n} x_{i}^{m_{i}}\right) F\left(x_{1}, \ldots, x_{n}\right)=0
$$

Communicated by H. Hironaka, October 14, 1986. Revised March 31, 1987.

* The research of this work was done with support of N.S.F.

** Mathematics Department, Purdue University, W. Lafayette, IN. 47907, U. S. A. 
satisfying the characteristic $p$ condition that the leading form of $\left(\prod_{i=1}^{n} x_{i}^{m_{i}}\right) F\left(x_{1}, \ldots, x_{n}\right)$ is not contained in $k\left[\left[x_{1}^{p^{e}}, \ldots, x_{n}^{p^{e}}\right]\right]$. The following proposition has been established by us and will be published elsewhere.

Proposition A. If ord $F\left(x_{1}, \ldots, x_{n}\right)=0$ then after finitely many blow-ups, the above singularity at the origin will have a smaller multiplicity.

However, it can be shown that ord $F\left(x_{1}, \ldots, x_{n}\right)$ may increase in general. This is a serious blow to the hope that ord $F\left(x_{1}, \ldots, x_{n}\right)$ will eventually drop to zero after monoidal transformations, and even open up the possibility that ord $F\left(x_{1}, \ldots, x_{n}\right)$ may increase indefinitely and thus a counter example to resolution may be constructed! It is the purpose of this article to establish the following theorem:

The Stability Theorem. Let $d=$ ord $F\left(x_{1}, \ldots, x_{n}\right)$. After a permissible blowup, along a residually rational valuation $v$ of the function field, say factor out $x_{1}$, and let $\tilde{F}=x_{1}^{-d} F$. Then ord $\tilde{F} \leq d+p^{e-1}$ and successive permissible blow-ups will not increase ord $\tilde{F}$ beyond the bound $d+p^{e-1}$ (in fact, $d+p^{r}$, see below) until it drops to $d$ or less.

We wish to express our deep thanks to Prof $\mathrm{H}$. Hironaka for his kindness and enlightening guidances on this important problem of mathematics.

\section{§1. A Proof of the Stability Theorem}

Let $k$ be an algebraically closed field of characteristic $p$ and $R=$ $k\left[\left[x_{1}, \ldots, x_{n}\right]\right]$, the power series ring of $n$ variables over $k$. Let

$$
z^{p^{e}}+\left(\Pi x_{i}^{m_{i}}\right) F\left(x_{1}, \ldots, x_{n}\right)=0
$$

be a purely inseparable equation to be resolved with $F\left(x_{1}, \ldots, x_{n}\right) \in R$, Let $d=\operatorname{ord} F\left(x_{1}, \ldots, x_{n}\right)$ and

$$
F\left(x_{1}, \ldots, x_{n}\right)=F_{d}\left(x_{1}, \ldots, x_{n}\right)+\text { higher terms. }
$$

If $m_{i} \geq p^{e}$ then we may replace $z$ by $z / x_{i}$ and cut down $m_{i}$ by $p^{e}$. Moreover, a translation of the form $z \rightarrow z+g$ will remove or change 
the $p^{e}$-th power part of $\left(\Pi x_{i}^{{ }}{ }^{i}\right) F_{d}\left(x_{1}, \ldots, x_{n}\right)$. We shall keep these two operations in mind.

Our basic assumption is $\left(\Pi x_{i}^{m_{i}}\right) F_{d} \notin R^{p^{e}}$ and $0 \leq m_{i}<p^{e}$. Let $v$ be any residually rational valuation given. We shall make the following definition and convention.

Definition. An ideal $P$ is said to be a permissible center if there is a system of parameters $\left\{y_{1}, \ldots, y_{n}\right\}$ such that (1) all $x_{i}{ }^{\prime}$ s with $m_{i} \neq 0$ are among them, (2) a part of them generate $P$, (3) $f \in P^{d}$ where $d=\operatorname{ord} F$. Note that if $P=\left(y_{1}, \ldots, y_{s}\right)$ then the leading form $F_{d}$ of $F$ is a homogeneous polynomial of degree $d$ in the leading form of $\left\{y_{1}, \ldots, y_{s}\right\}$. We assume that $\left\{y_{1}, \ldots, y_{n}\right\}$ is $\left\{x_{1}, \ldots, x_{n}\right\}$ 。

Convention. After making a choice of the order of $n$ variables as $x_{1}, \ldots, x_{n}$, then in the monoidal transformation we always factor out the $x_{i}$ satisfying the following conditions: (1) $x_{i}$ is in the center $P$ of the permissible monoidal transformation; (2) $v\left(x_{i}\right)=\min \{v(\alpha)$ : $\alpha \in P\}$; (3) the integer $i$ is the minimal integer satisfying condition (2). With such an $x_{t}$, the monoidal transformation will be of the following form:

$$
\begin{aligned}
x_{i}=\bar{x}_{i} & \\
x_{j}=\bar{x}_{i} \bar{x}_{j} & \forall j<i, x_{j} \in P \\
x_{k}=\bar{x}_{i}\left(\bar{x}_{k}+\alpha_{k}\right) & \forall k>i, x_{k} \in P \\
x_{l}=\bar{x}_{l} & \forall x_{l} \notin P
\end{aligned}
$$

where $\alpha_{k} \in k$.

To simplify our notation we may assume that $i=1$, namely, $x_{1} \in P$ and

$$
v\left(x_{1}\right) \leq v\left(x_{j}\right) \quad \forall x_{j} \in P .
$$

Moreover we shall use the subdivision of the set of variables $\left\{x_{1}, \ldots\right.$, $\left.x_{n}\right\}=\left\{x_{1}\right\} \cup X \cup Y \cup Z$ where

$$
\begin{aligned}
& X=\left\{x_{i}: x_{i} \in P,{ }_{i} \neq 1, m_{i}=0 \text { or } v\left(x_{i}\right)>v\left(x_{1}\right)\right\} \\
& Y=\left\{x_{j}: x_{j} \in P,{ }_{j} \neq 1, m_{j} \neq 0 \text { and } v\left(x_{j}\right)=v\left(x_{1}\right)\right\} \\
& Z=\left\{x_{l}: x_{l} \notin P\right\} .
\end{aligned}
$$

Note that for $x_{i} \in X$, if $v\left(x_{i}\right)=v\left(x_{1}\right)$, then we may let $x_{i}^{*}=x_{i}+\alpha_{i} x_{1}$ with $v\left(x_{i}^{*}\right)>v\left(x_{1}\right)$. Since the corresponding $m_{i}=0$ then such a translation 
will not change the form of $\Pi_{x_{i}}^{m_{i}} F\left(x_{1}, \ldots, x_{n}\right)$ i. e., it will not affect the basic condition that $\Pi x_{i}^{m} \cdot F_{d}\left(x_{1}, \ldots, x_{n}\right) \notin R^{p^{e}}$. So we may assume $v\left(x_{i}\right)>v\left(x_{1}\right)$ for all $x_{i} \in X$. Note that $F_{d}\left(x_{1}, \ldots, x_{n}\right)$ is independent of $x_{l}, \forall x_{l} \notin Z$ because $P$ is a permissible center.

Thus the monoidal transformation will be of the following form

$$
\begin{aligned}
x_{1}=\bar{x}_{1} & \\
x_{i}=\bar{x}_{1} \bar{x}_{i} & \forall x_{i} \in X \\
x_{j}=\bar{x}_{1}\left(\bar{x}_{j}+\alpha_{j}\right) & \forall x_{j} \in Y \\
x_{l}=\bar{x}_{l} & \forall x_{l} \in Z
\end{aligned}
$$

where $0 \neq \alpha_{j} \in k$. For the following discussions we will introduce Hasse derivations $\left\{d_{y}^{(a)}\right\}$ as

Definition. Let $S$ be a commutative ring, $f(y)$ in $S[[y]]$ and $f(y+t) \in$ $S[[y, t]]$. In the expansion

$$
f(y+t)=f(y)+\sum_{a \geq 1} f^{(a)}(y) t^{a}
$$

the operation $d_{y}^{(a)}(f(y))$ is defined to be $f^{(a)}(y)$.

The following proposition is easy.

\section{Proposition 1. We have}

(1) $d_{y}^{(1)}$ is the usual derivation and $d_{y}^{(a)}$ is linear over $S$.

(2) $f(y) \in S\left[\left[y^{p^{r}}\right]\right] \backslash S\left[\left[y^{p^{r}+1}\right]\right] \Leftrightarrow d_{y}^{(1)}(f(y))=\ldots=d_{y}^{\left(p^{r}-1\right)}(f(y))=0$ and $d_{y}^{\left(p^{r}\right)}(f(y)) \neq 0$.

(3) $d_{y}^{\left(p^{r}\right)}$ is a nonzero derivation on $S\left[\left[y^{p^{r}}\right]\right]$.

Proof: We only prove (2), the rest being easy. Note that $d_{y}^{(j)}\left(y^{s}\right)$ $=C_{s, j} y^{s-j}$ where $C_{s, j}$ is a binomial coefficient. For the part $\Rightarrow$, it follows from binomial expansion that $D_{y}^{(1)}(f(y))=\ldots=d^{\left(p^{r}-1\right)}(f(y))=0$. Let $s$ be the minimal integer such that $a_{s} y^{s p^{r}}$ appears in $f(y)$ where $a_{s} \neq 0$ and $p \nmid s$. Then $d_{y}^{\left(p^{r}\right)}\left(a_{s} y^{s p^{r}}\right)=s a_{s} y^{(s-1) p^{r}} \neq 0$ and other terms are either zero or with exponents $>(s-1) p^{r}$. Thus $d_{y}^{\left(p^{r}\right)}(f(y)) \neq 0$. On the other hand, for the part $\Leftarrow$, let

$$
f(y) \in S\left[\left[{y^{p}}^{p^{1}}\right]\right] \backslash S\left[\left[y^{p^{r_{1}+1}}\right]\right]
$$

for some $r_{1}$. It is easy to see $r_{1}=r$ by what we just proved.

Q. E. D. 
Proposition 2. Let $k$ be a field of characteristic $p, y^{m} \varphi(y) \in k\left[y^{p^{r}}\right] \backslash$ $k\left[y^{p^{r+1}}\right]$ and $0 \neq \alpha \in k$. Let $\operatorname{deg} \varphi(y)=d^{*},(y+\alpha)^{m} \varphi(y+\alpha)=\sum a_{i} y^{i}, c=$ $\min \left\{i: a_{i}=0, p^{r+1} \nmid i\right\}$. Then $c \leq d^{*}+p^{r}$.

Proof: Let $\varphi(y)=y^{n} \circ \varphi^{*}(y)$ with $\varphi^{*}(0) \neq 0$. Then we have $p^{r} \mid$ $(m+n)$. Without losing gernality we may assume $n=0, p^{r} \mid m$, and $\varphi(y) \in k\left[y^{p^{r}}\right]$. It follows from our Proposition 1 that $d^{\left(p^{r}\right)}$ is a derivation on $k\left[y^{p^{r}}\right]$. Thus we get

and

$$
d_{y}^{\left(y^{r}\right)}=d_{y+\alpha}^{\left(v^{r}\right)} \text { on } k\left[y^{p^{r}}\right]
$$

$$
(y+\alpha)^{m-p^{r}}, y^{c-p^{r}} \mid d_{y}^{\left(p^{r}\right)}\left((y+\alpha)^{m} \varphi(y+\alpha)\right) .
$$

Moreover, the right hand side is a polynomial of degree $\leq d^{*}+m-p^{r}$. Since $\alpha \neq 0,(y+\alpha)^{m-p^{r}}$ and $y^{c-p^{r}}$ and are coprime. Then we have

$$
m-p^{r}+c-p^{r} \leq d^{*}+m-p^{r}
$$

or

$$
c \leq d^{*}+p^{r}
$$

Q.E.D.

The blow-up with a permissible center $P$ of $f\left(x_{1}, \ldots, x_{n}\right)$ will transform $\left(\Pi x_{i}^{m_{i}}\right) F\left(x_{1}, \ldots, x_{n}\right)$ to the following

$$
\bar{x}_{1}^{m_{1}} \prod_{x_{i} \in X} \bar{x}_{i}^{m_{i}} \cdot \prod_{x_{j} \in Y}\left(\bar{x}_{j}+\alpha_{j}\right)^{m_{j}} \prod_{x_{l} \in Z} \bar{x}_{l}^{m_{l}}\left(F_{d}\left(1, \ldots, \bar{x}_{2}, \ldots, \bar{x}_{j}+\alpha_{j}, \ldots,\right)+g\right)
$$

where $\bar{m}_{1}=m_{1}+\sum_{x_{i} \in X} m_{i}+\sum_{x_{j} \in Y} m_{j}$ and $g \in I=$ the ideal generated by $\left\{\bar{x}_{1}, \bar{x}_{l}\right.$ : $\left.x_{l} \in Z\right\}$. Suppose $\bar{m}_{1} \not \equiv 0\left(p^{e}\right)$. Then in the product

$$
\prod_{x_{j} \in Y}\left(\bar{x}_{j}+\alpha_{j}\right)^{m_{j}} \cdot\left(F_{d}\left(1, \ldots, \bar{x}_{i}, \ldots, \bar{x}_{j}+\alpha_{j}, \ldots\right)+g\right)
$$

we consider the terms which do not involve $\bar{x}_{1}$ and $\bar{x}_{l}$ for all $x_{l} \in Z$. They will not be cancelled by terms in $g$ and have order $\leq d$ in $\bar{x}_{\imath}$ 's and $\bar{j}$ s.

Hence we may rewrite the transform of $\prod_{x_{i}}^{m_{i}} F\left(x_{1}, \ldots, x_{n}\right)$ after throwing away $p^{e}$-th power terms as

$$
\bar{x}_{1}^{n_{1}} \prod_{x_{i} \in X} \bar{x}_{i}^{m_{i}} \prod_{x_{l} \in Z} \bar{x}_{l}^{m_{l}}\left(\tilde{F}_{\tilde{d}}\left(\bar{x}_{1}, \ldots, \bar{x}_{n}\right)+\text { higher terms }\right) .
$$

Naturally we have $\tilde{d} \leq d$. Thus our stability theorem is proved in 
the case that $\bar{m}_{1} \not \equiv 0\left(p^{e}\right)$. For our convenience we shall call this case Possibility (I).

From now on, let us assume $\bar{m}_{1} \equiv 0\left(p^{e}\right)$. Due to our basic assumption that

$$
\Pi x_{i}^{m_{i}} F_{d}\left(x_{1}, \ldots, x_{n}\right) \notin R^{p^{e}}=k\left[\left[x_{1}^{p^{e}}, \ldots, x_{n}^{p^{e}}\right]\right]
$$

and $\bar{m}_{1} \equiv 0\left(p^{e}\right)$ it follows that

$$
\begin{aligned}
\prod_{x_{i} \in X} \bar{x}_{i}^{m_{i}} \prod_{x_{j} \in Y}\left(\bar{x}_{j}+\alpha_{j}\right)^{m_{j}} \prod_{x_{l} \in Z} \bar{x}_{l}^{m_{l}} F_{d}\left(1, \ldots, \bar{x}_{i}, \ldots, \bar{x}_{j+d_{j}}, \ldots\right) \\
\quad \notin k\left[\ldots, \bar{x}_{i}^{p^{e}}, \ldots,\left(\bar{x}_{j}+\alpha_{j}\right)^{p^{e}}, \ldots, \bar{x}_{l}^{p^{e}}, \ldots,\right] \\
\quad=k\left[\bar{x}_{2}^{p^{e}}, \ldots, \bar{x}_{i}^{p^{e}}, \ldots, \bar{x}_{j}^{p^{e}}, \ldots, \bar{x}_{l}^{p^{e}}, \ldots\right]
\end{aligned}
$$

So there is an $\bar{x}_{s}$ such that

$$
\begin{gathered}
\Pi \bar{x}_{i}^{m_{i}} \Pi\left(\bar{x}_{j}+\alpha_{j}\right)^{m_{j}} \Pi \bar{x}_{l}^{m_{l}} F_{d}\left(1, \ldots, \bar{x}_{i}, \ldots, \bar{x}_{j}+\alpha_{j}, \ldots\right) \\
\notin \notin k\left[\bar{x}_{2}, \ldots, \hat{\bar{x}}_{s}, \ldots, \bar{x}_{j}, \ldots\right]\left[\bar{x}_{s}^{p^{e}}\right] .
\end{gathered}
$$

If $\bar{x}_{s}=\bar{x}_{l}$ for some $x_{l} \in Z$, i. e. $m_{l} \not \equiv 0\left(p^{e}\right)$ then clearly the leading form of $\Pi \bar{x}_{i}^{m_{i}} \Pi\left(\bar{x}_{j}+\alpha_{j}\right)^{m_{j}} \Pi \bar{x}_{l}^{m_{l}} F_{d}$ coincides with $\Pi \bar{x}_{i}^{m_{i}} \Pi \bar{x}_{l}^{m_{l}}$ times the leading form of $\left(\Pi\left(\bar{x}_{j}+\alpha_{j}\right)^{m_{j}} F_{d}\right)$, which is not in $k\left[\bar{x}_{2}^{p^{e}}, \ldots, x_{j}^{p^{e}}, \ldots\right]$ and ord $\Pi\left(\bar{x}_{j}+\alpha_{j}\right)^{m_{j}} F_{d}\left(1, \ldots, \bar{x}_{i}, \ldots, \bar{x}_{j}+\alpha_{j}, \ldots\right) \leq d$. Thus our theorem is proved in this case. We shall call this case Possibility (II). Henceforth we may assume that $m_{l} \equiv 0\left(p^{e}\right)$ for all $x_{l} \in Z$.

Let $r$ be the nonnegative integer such that $\Pi x_{i}^{m_{i}} F_{d}\left(x_{1}, \ldots, x_{n}\right) \in$ $R^{p^{r}} \backslash r^{p^{r+1}}$. Then clearly its transformation belongs to $\left(k\left[\bar{x}_{1}, \ldots, \bar{x}_{n}\right]\right)^{p^{r}} \backslash$ $\left(k\left[\bar{x}_{1}, \ldots, \bar{x}_{n}\right]\right)^{p^{r+1}}$.

Note that $\Pi_{x_{i}}^{m_{i}} F_{d}\left(x_{1}, \ldots, x_{n}\right) \in k\left[x_{1}^{p^{r}}, \ldots, x_{n}^{p^{r}}\right]$. If $p^{r} \nmid m_{i}$, then we may factor out more $x_{i}$ from $F_{d}\left(x_{1}, \ldots, x_{n}\right)$. So, if necessary, we may assume that

$$
p^{r} \mid d=\operatorname{ord} F_{d}\left(x_{1}, \ldots, x_{n}\right)
$$

Remark: In other words, ord $F_{d}\left(x_{1}, \ldots, x_{n}\right)$ may be taken to be less than or equal to $\left[d / p^{r}\right] p^{r}$ in the numerical discussions below.

We shall assume that for a particular $\bar{x}_{s}$

$$
\begin{gathered}
\Pi \bar{x}_{i}^{m_{i}} \Pi\left(\bar{x}_{j}+\alpha_{j}\right)^{m_{j}} \Pi \bar{x}_{l}^{m_{l}} F_{d}\left(1, \ldots, \bar{x}_{2}, \ldots, \bar{x}_{j}+\alpha_{j}, \ldots\right) \\
\notin k\left[\bar{x}_{2}, \ldots, \hat{x}_{s}, \ldots, \bar{x}_{j}, \ldots\right]\left[\bar{x}_{s}^{p^{r+1}}\right] .
\end{gathered}
$$

For the remainder of the proof we will consider two possibilities; 
(III) $\bar{x}_{s} \in X$ or (IV) $\bar{x}_{s} \in Y$.

Possibility (III): Note that

$$
\begin{gathered}
\Pi x_{i}^{m_{i}} \Pi x_{j}^{m_{j}} F_{d}\left(x_{1}, \ldots, x_{i}, \ldots, x_{j}, \ldots\right) \\
\notin k\left[x_{1}, \ldots, \hat{x}_{s}, \ldots, x_{j}, \ldots\right]\left[x_{s}^{p^{r+1}}\right] \Leftrightarrow \Pi \bar{x}_{i}^{m_{i}} \Pi\left(\bar{x}_{j}+\alpha_{j}\right)^{m_{j}} F_{d}(1, \ldots, \\
\left.\bar{x}_{i}, \ldots, \bar{x}_{j}+\alpha_{j}, \ldots\right) \notin k\left[\bar{x}_{2}, \ldots, \hat{x}_{s}, \ldots, \bar{x}_{j}, \ldots\right]\left[\bar{x}_{s}^{p^{r+1}}\right] .
\end{gathered}
$$

Let

$$
\Pi \bar{x}_{i}^{m_{i}+n}\left(h\left(\left\{\bar{x}_{j}\right\}\right)\right)
$$

be a term in the expansion of $\Pi \bar{x}_{i}^{m_{i}} F_{d}\left(1, \ldots, \bar{x}_{i}, \ldots, \bar{x}_{j}+\alpha_{j}, \ldots\right)$ with $\bar{x}_{i} \in X$ as variables and $k\left[\left\{\bar{x}_{j} ; x_{j} \in Y\right\}\right]$ as coefficients. Note $p^{r+1} \nmid$ $\left(m_{s}+n_{s}\right)$. Then clearly we have

$$
\text { ord } h \Pi\left(\bar{x}_{j}+\alpha_{j}\right)^{m_{i}} \leq d-\sum n_{\imath} \text { 。 }
$$

Thus we have established the non-increase of the order of $F$. Note that in all previous discussions the order of $F$ will not increase.

Now let us recall Proposition 2 for the discussion of Possibility (IV). In the following expression let $\bar{y}_{s}=\bar{x}_{s}+\alpha_{s}$.

$$
\begin{aligned}
& \left(\bar{x}_{s}+\alpha_{a}\right)^{m_{s}} F_{d}\left(1, \ldots, \bar{x}_{i}, \ldots, \bar{x}_{j}+\alpha_{j}, \ldots\right) \\
= & \bar{y}_{s}^{m_{s}} F_{d}\left(1, \ldots, \bar{x}_{i}, \ldots, \bar{x}_{j}+\alpha_{j}, \bar{y}_{s}, \ldots\right) \\
= & \sum \bar{y}_{s}^{m_{s}} g_{I}\left(\bar{y}_{s}\right) \Pi_{j \neq s} \bar{x}_{j}^{n_{j}}
\end{aligned}
$$

where $I=\left(n_{1}, \ldots, n_{j}, \ldots\right)$ and $g_{I}\left(\bar{y}_{s}\right)$ is a polynomial in $\bar{y}_{s}$. We have at least one $I$ such that

$$
\bar{y}_{s}^{m_{s}} g_{I}\left(\bar{y}_{s}\right) \in k\left[\bar{y}_{s}^{p^{r}}\right] \backslash k\left[\bar{y}_{s}^{p^{r+1}}\right] .
$$

Say, $\bar{y}_{s}^{m_{s}} g_{I}\left(\bar{y}_{s}\right) \in k\left[\bar{y}^{p^{r}}\right] \backslash k\left[\bar{y}^{p^{r+1}}\right]$. Moreover we have

$$
\operatorname{deg} g_{I}\left(\bar{y}_{s}\right)+|I| \leq d \text { (in fact, }\left[d / p^{r}\right] p^{r} \text {. See Remark) }
$$

where $|I|=\sum n_{j}$. Now make the substitution $\bar{y}_{s}=\bar{x}_{s}+\alpha_{s}$ and expand the polynomial. It follows from Proposition 2 that in the expansion there is a term $\bar{x}_{s}^{c}$ such that

$$
p^{r+1} \nmid c, c \leq \operatorname{deg} g_{I}\left(\bar{y}_{s}\right)+p^{r} \leq d-|I|+p^{r} .
$$

Moreover, it is easy to see that

(1) the total degree of $\bar{x}_{x}^{c} \cdot \Pi \bar{x}_{j}^{n_{j}}$ is at most $d+p^{r}$ (in fact, $\left[d / p^{r}\right]$. $p^{r}+p^{r}$. See Remark.)

(2) $c \geq p^{r}, p^{r+1} \nmid c$ 。 
Now we shall collect the polynomial in terms of $\bar{x}_{\mathrm{s}}$ as follows :

$$
\begin{aligned}
& \left(\bar{x}_{s}+\alpha_{s}\right)^{m_{s}} F_{d}\left(1, \ldots, \bar{x}_{j}+\alpha_{j}, \ldots\right) \\
& \quad=\sum h_{i}\left(\ldots, \bar{x}_{i}, \ldots, \bar{x}_{j}, \ldots\right) \bar{x}_{s}^{i} .
\end{aligned}
$$

Then we have by (1) that

(3) ord $h_{c} \tilde{x}_{s}^{c} \leq d+p^{r}$ (in fact, [d/p $\left.p^{r}\right] p^{r}+p^{r}$. See Remark).

Now multiplying it with the remaining $\left(\bar{x}_{j}+\alpha_{j}\right)^{m_{j}}$, we conclude easily that

$$
\text { ord } \prod_{j \neq s}\left(\bar{x}_{j}+\alpha_{j}\right)^{m_{j}} h_{c} \bar{x}_{s}^{c} \leq d+p^{r} \text {. }
$$

Hence we have the following statement.

Statement: In the possibility (IV) after we blow-up the permissible center $P$, let ord $\tilde{F}=d_{1}$, then $\tilde{F}$ has a term $A$ with

(i) ord $A \leq d+p^{r}$ (in fact, $\left[d / p^{r}\right] \cdot p^{r}+p^{r}$. See Remark)

(ii) $\operatorname{ord}_{\bar{x}_{s}} A=c \geq p^{r}$ and $p^{r+1} \nmid c$

(iii) $d_{1} \leq$ ord $A \leq d+p^{r}$ (in fact, $\left[d / p^{r}\right] p^{r}+p^{r}$. See Remark).

The interesting thing is that now $\bar{x}_{s}$ is an $X$-kind of variable due to the fact that $m_{s}$ becomes zero. Furthermore, we shall use our Convention and call $\bar{x}_{s}$ the last variable.

Let us assume that $d_{1}=\operatorname{ord} A=d+p^{r}$. We may request that $c$ is the largest one satisfying conditions (i), (ii) and (iii) in the leading form of $\tilde{F}$. Let us examine the further blow-ups. There are two cases: (1) $v\left(\bar{x}_{s}\right)$ is the only minimal. (2) $v\left(\bar{x}_{s}\right)$ is not the only minimal. In the first case, we have to factor out $\bar{x}_{s}$ and do it without any translation. Due to the existence of the term $A$, the order of $\tilde{F}$ will drop at least by $c$ which is $\geq p^{r}$. Hence the order of $\tilde{F}$ will drop to $d^{\prime} \leq d$. Our proposition is proved in this case.

In the second case, we simply note that if by factoring out $\bar{x}_{1}$ (which is not $\bar{x}_{s}$ ) and then translating (i. e., replace $\bar{x}_{s}$ by $\bar{x}_{1}\left(\overline{\bar{x}}_{x}+\alpha_{s}\right)$ ) the order of $\tilde{F}$ will not increase (c. f. Possibilities (I), (II) or (III))。 If the order of $\tilde{F}$ drops by further blow-ups, we may assume that $d_{1}=$ ord $\tilde{F}<\left[d / p^{r}\right] \cdot p^{r}+p^{r}$ from the very beginning.

Let us assume $d<d_{1}$. Then the following inequality

$$
d<d_{1}<\left[d / p^{r}\right] p^{r}+p^{r}
$$

implies 


$$
p^{r} \nmid d_{1} \text {. }
$$

Let $r_{1}$ be defined by

$$
\begin{gathered}
p^{r_{1}} \mid d_{1} \\
p^{r_{1}+1} \nmid d_{1} .
\end{gathered}
$$

Then $r_{1}<r$. We conclude easily that the new bound for ord $\tilde{F}$ after blow-ups will be

$$
d_{1}+p^{r_{1}}<d+p^{r}
$$

Repeating the above argument, we establish that $d+p^{r}$ is the upper bound for orders for all successive blow-ups until the order becomes less than or equal to $d$.

Q. E. D.

\section{References}

[AM1] S.S. AbHYANKAR AND T. T. MOH, Newton-Puiseux expansion and generalized Tschirnhausen transformation I, Journal für die reine und angewandte Mathematik, 260 (1973), 47-83.

[AM2] - Newton-Puiseux expansion and generalized Tschirnhausen transformation, II, Journal für die reine und angewandte Mathematik, 261 (1973), 29-53.

[H] H. HironaKA, Idealistic exponents of singularity, "Algebraic Geometry," Johns Hopkins Univ. Press, 1977.

[M] T. T. MOH, On two fundamental theorems for the concept of approximate roots, J. Japan Math. Soc., 34:4 (1982), 637-652. 
